\title{
ON CARLEMAN INTEGRAL OPERATORS
}

GYÖRGY I. TARGONSKI

1. Introduction. Integral operators on the Hilbert function space $L^{2}(a, b)$

$$
(K f)(x)=\int_{a}^{b} k(x, y) f(y) d y \quad \text { for all } f \in L^{2}(a, b)
$$

with the property

$$
\int_{a}^{b}|k(x, y)|^{2} d y<\infty \quad \text { for a.a. } x
$$

were originally defined by T. Carleman [1]; he imposed on the kernel the condition of hermiticity, and also demanded

$$
\lim _{x \rightarrow x} \int_{a}^{b}\left|K\left(x^{\prime}, y\right)-K(x, y)\right|^{2} d y=0
$$

for all $x$ with exception of a countable set with a finite number of limit points, and

$$
\int_{J_{\delta}}\left[\int_{a}^{b}|k(x, y)|^{2} d y\right] d x<\infty, \quad \text { for every } \delta>0
$$

where $J_{\delta}$ denotes the interval $[a, b]$ with exception of the subintervals $\left|x-x_{n}\right|<\delta$; here $x_{n}$ denotes a finite set of points, chosen from among the points for which (3) does not hold.

The question, whether a given operator can be represented in the form (1), with condition (2), was investigated by J. von Neumann [2]; he dropped conditions (3) and (4), but essentially restricted the investigation to the case of hermitean operators. von Neumann's work was based on previous results by $\mathrm{H}$. Weyl [3].

More recently, the results of von Neumann were generalized to nonhermitean, especially normal operators and various other results were derived, with a view to application to scattering theory in quantum physics [4].

The present paper describes additional results in this direction.

We shall start with several definitions. By "operator" we shall mean a not necessarily bounded linear transformation on the Hilbert function space $L^{2}(a, b)$ of complex-valued functions of one real vari-

Received by the editors July 12, 1966. 
able, where $-\infty \leqq a<b \leqq \infty$. We shall also use the Hilbert function space $L^{2}(a, b)$ of complex-valued functions of two real variables, defined on the square $a \leqq x \leqq b, a \leqq y \leqq b$.

Definition 1. $K$ is a Carleman integral operator, if it can be represented in the form (1) with condition (2) satisfied. We do not demand that (3) and (4) be satisfied.

Definition 2. $K$ is an operator of Carleman integral type, if it is unitarily equivalent to a Carleman integral operator, i.e. if a unitary operator $U$ exists such that $U K U^{*}$ is a Carleman integral operator.

Definition 3. $K$ is a strong Carleman integral operator if $U K U^{*}$ is a Carleman integral operator for every unitary $U$.

Definition 4. $\lambda_{0}$ is a Weyl limit point of the spectrum of a normal operator, if at least one of the following conditions is satisfied.

(a) $\lambda_{0}$ is a discrete eigenvalue with infinitely high multiplicity.

(b) $\lambda_{0}$ is the limit of a sequence of discrete eigenvalues.

(c) $\lambda_{0}$ belongs to the continuous spectrum.

Definition 5. A (not necessarily normal) operator $\Omega$ is a HilbertSchmidt operator, if

$$
\sum_{n}\left\|\Omega \phi_{n}\right\|^{2}<\infty
$$

where $\left\{\phi_{n}\right\}$ is any complete orthonormal set in $L^{2}(a, b)$. The sum is independent of the choice of $\left\{\phi_{n}\right\}$. We now summarize a few known results.

I ([4], for the selfadjoint case [2]). A normal operator (defined by $N N^{*}=N^{*} N$ ) is of Carleman integral type if and only if zero is a Weyl limit point of its spectrum.

II ([4]). A bounded operator is a strong Carleman operator if and only if it is a Hilbert-Schmidt operator. (It is not known, whether unbounded strong Carleman integral operators exist.)

III ([4]). If $K$ is a Carleman integral operator, and $B$ is a bounded operator, then $K B$ is a Carleman integral operator, and if $K$ is represented by the kernel $k(x, y)$, then $K B$ is represented by the kernel

$$
k(K B)=C B^{*} C k(x, y)
$$

where $C$ stands for complex conjugation and $B^{*}$ acts on $k(x, y)$ as on a function of $y$, while $x$ is considered a fixed parameter.

IV ([4]). The class of bounded, strong Carleman integral operators is a two sided ideal in the algebra of bounded operators.

V. The adjoint of a (not necessarily bounded) normal Carleman integral operator is again a normal Carleman integral operator.

For a more detailed account of the theory and for several related results we refer to $[4]$ and $[6]$. 
2. Operators with "locally bounded" range.

DeFinition 6. A bounded operator $A$ is of locally bounded range if on a number $M$ exists, independent of $f$ and $x$, such that

$$
|(A f)(x)| \leqq M\|f\| \quad \text { for all } f \in L^{2}(a, b)
$$

and for every $a \leqq x \leqq b$.

The class of operators with locally bounded range is certainly not empty, as the example of the zero operator shows. To construct a nontrivial example, we choose a function $\psi(x) \in L^{2}(a, b)$ with the property $\|\psi(x)\|=1,|\psi(x)| \leqq M$ and define $A$ by

$$
(A f)(x)=\int_{a}^{b} \psi(x) \Psi(y) f(y) d y .
$$

The operator $A$ is now a projection operator on a one-dimensional subspace; it is a selfadjoint Hilbert-Schmidt operator. One certainly has

$$
\begin{aligned}
|(A f)(x)| & =\left|\psi(x) \int_{a}^{b} \bar{\psi}(y) f(y) d y\right| \leqq|\psi(x)| \cdot\|\Psi\| \cdot\|f\| \\
& =|\psi(x)| \cdot\|f\| \leqq M\|f\| .
\end{aligned}
$$

$A$ is therefore an operator of locally bounded range in the sense of Definition 6. We now give a necessary condition for an operator to have a locally bounded range.

LEMma 1. If an operator is of locally bounded range, then it is a Carleman integral operator; and if it is normal, zero is a Weyl limit point of its spectrum.

Before proving Lemma 1, we point out that the condition cannot be sufficient; it is easy to construct a Carleman integral operator the range of which is not locally bounded. Proceeding on the lines of our previous example for the existence of a nonzero operator with locally bounded range, we replace $\psi(x)$ by an unbounded $L^{2}$-function $\Psi(x)$; then all $f$ which are nonorthogonal to $\Psi$ are transformed by $A$ into multiples of $\Psi$, i.e. locally unbounded functions. $A$ however is now still a Hilbert-Schmidt operator, a fortiori a Carleman integral operator.

To obtain the proof of Lemma 1, we introduce the linear functional $L\left(x_{0}\right)$ by

$$
L\left(x_{0}\right) f=(A f)(x)_{x=x_{0}} .
$$

Since $A$ is bounded, $L$ is defined for all $f \in L^{2}(a, b)$, it is linear and because of (7), bounded. Therefore, according to the lemma of 
F. Riesz, there exists a $\alpha_{x_{0}} \in L^{2}(a, b)$, such that

$$
L\left(x_{0}\right) f=\left[\alpha_{x_{0}}, f\right] \text {. }
$$

Since (11) holds for all $a \leqq x_{0} \leqq b$, we can write

$$
L(x) f=(A f)(x)=\left[\alpha_{x}, f\right]=\int_{a}^{b} \alpha(x, y) f(y) d y
$$

where $\alpha(x, y)=\alpha_{x}(y) \in L^{2}(a, b)$, therefore (2) is satisfied. For normal operators, Lemma 1 implies according to $I$, that $A$ contains zero as a Weyl limit point in the spectrum. This completes the proof.

3. Strong Carleman integral operators. According to Definition 3, if $K$ is a strong Carleman integral operator, then so is $U K U^{*}$ for every unitary operator $U$; also, IV states that the class of bounded, strong Carleman integral operators is a two sided ideal in the algebra of bounded operators. Actually somewhat more can be said; if unbounded, strong Carleman integral operators exist, the class of not necessarily bounded strong Carleman integral operators is closed under multiplication by arbitrary bounded operators from the right, or from the left.

We shall however restrict ourselves to the class of bounded, strong Carleman integral operators, i.e. the Hilbert-Schmidt class (of in general not normal operators) as determined by Definition 5 . The question we pose is the following: What (obviously linear) transformation does the kernel undergo, when the corresponding operator is multiplied by bounded operators from the left, or from the right, or generally from both sides (this case including the case of unitary equivalence)? We introduce the notation $k(X)$ for the kernel belonging to a Hilbert-Schmidt operator $X$. By definition, one has

$$
\int_{a}^{b} \int_{a}^{b}|k(\Omega)|^{2} d x d y<\infty
$$

if and only if $\Omega$ is a Hilbert-Schmidt operator in the general sense of Definition 5.

We set out to determine $k(\Omega B), k(A \Omega)$, and $k\left(A \Omega_{\iota} B\right)$, in particular $k\left(U A U^{*}\right)$, where $A$ and $B$ shall denote bounded operators, and $U$ an unitary operator. From III, we can rewrite (6) in the for $\mathrm{m}$

$$
k(\Omega B)=C B^{*} C k(\Omega) .
$$

Moreover, (cf. [5, pp. 227 and 231]) one has

$$
k\left(\Omega^{*}\right)=\operatorname{ECk}(\Omega)
$$


This relation does not hold for Carleman kernels in general, as one can easily see constructing a kernel $k(x, y)$ where the Carleman property (2) holds, but does not hold for the kernel $\vec{k}(y, x)$; on the other hand, (15) is true not only for Hilbert-Schmidt kernels, but also for the wider class of kernels which possess the Zaanen $P$ property (see [5]).

It follows from IV, that

$$
\int_{a}^{b} \int_{a}^{b}|k(A \Omega)|^{2} d x d y<\infty
$$

and

$$
\int_{a}^{b} \int_{a}^{b}|k(\Omega B)|^{2} d x d y<\infty
$$

hold, if $A$ and $B$ are bounded and (13) holds. To find $k(A \Omega)$, we combine (15) and (14) and find

$$
\begin{aligned}
k(A \Omega) & =k\left[\left(\Omega^{*} A^{*}\right)^{*}\right]=\operatorname{ECk}\left(\Omega^{*} A^{*}\right) \\
& =\operatorname{ECCACk}\left(\Omega^{*}\right)=\operatorname{ECCACECk}(\Omega)=\operatorname{EA} \operatorname{Ek}(\Omega) .
\end{aligned}
$$

We can now settle the general case using (14) and (18):

$$
\begin{aligned}
k(A \Omega B) & =k[(A \Omega) B]=C B^{*} C k(A \Omega) \\
& =C B^{*} C E A E k(\Omega)=C B^{*} E C A E k(\Omega) ;
\end{aligned}
$$

alternatively,

$$
\begin{aligned}
k(A \Omega B) & =k[A(\Omega B)]=E A E k(\Omega B) \\
& =E A E C B^{*} C k(\Omega)=E A C E B^{*} C k(\Omega) .
\end{aligned}
$$

For the case of unitary equivalence, $A=U, B=U^{*}$, (19) and (20) become

$$
k\left(U \Omega U^{*}\right)=(C U E)^{2} k(\Omega)=(E U C)^{2} k(\Omega) .
$$

(19) and (20) can be interpreted in a slightly different manner: Let us now put $k(\Omega)=\omega(x, y)$ and remark that $\omega(x, y)$ satisfies the relations

$$
\begin{array}{ll}
\omega(x, y) \in L^{2}, & \\
\omega(x, y) \in L^{2} & \text { for a.a. fixed } x, \\
\omega(x, y) \in L^{2} & \text { for a.a. fixed } y .
\end{array}
$$

We shall denote the second and third lines in (22) by 


$$
\omega(x, y) \in L^{2}(y) \text { and } \omega(x, y) \in L^{2}(x), \text { respectively. }
$$

If now $A$ and $B$ are bounded operators on $L^{2}$, we can define the operator $A_{x}, A_{x} \omega(x, y)$ meaning that $A$ acts on $\omega$ as on a function of $x$, while $y$ remains fixed; in an analogous manner, we can define $B_{y}$. It has to be noted that, so far, we interpreted $B$ as $B_{y}$ (see III).

Using this notation, one has

$$
E A E=E A_{y} E=A_{x}
$$

and thus (19) and (20) yield, without any reference to the problem of determining $k(A \Omega B)$, and taking into consideration $E C=C E$.

$$
C B_{y}^{*} C A_{x}=A_{x} C B_{y}^{*} C .
$$

Replacing $B^{*}$ by $B$ and considering a real space $L^{2}$, one has

$$
B_{y} A_{x}=A_{x} B_{y},
$$

a result analogous to Young's theorem about interchangeability of partial derivations.

Let us summarize our findings in

LeMma 2. Let $\omega \in L^{2}$ be an element of a Hilbert space of functions of two variables and $A, B$ bounded operators on the Hilbert space $L^{2}$ of functions of one variable, $A_{x} \omega$ and $B_{y} \omega$ denoting that $A$ acts on $\omega$ as on a function of $x$ and $B$ acts on $\omega$ as on a function of $y$, then

$$
C B_{y} C A_{x} \omega=A_{x} C B_{y} C \omega \quad \text { for all } \omega \in L^{2}
$$

where $C$ denotes complex conjugation; and, if $\omega$ is interpreted as the kernel belonging to a (generalized) Hilbert-Schmidt transformation, then $C B_{y} C A_{x} \omega(x, y)=A_{x} C B_{y} C \omega(x, y)$ is the kernel belonging to the HilbertSchmidt operator $A \Omega B^{*}$; in particular,

$$
C U_{y} C U_{x} \omega(x, y)=U_{x} C U_{y} C \omega(x, y)
$$

is the kernel belonging to the unitary equivalents $U \Omega U^{*}$.

Therefore the following relationships hold:

$$
A_{x} C B_{y} C \omega \in L^{2} \quad \text { if } \omega \in L^{2} ;
$$

and choosing $B_{y}=I$

$$
A_{x^{\omega}} \in L^{2}
$$

and symmetrically for $A_{x}=I C B_{y} C \omega \in L^{2}$; therefore,

$$
B_{y} \omega \in L^{2} \quad \text { if } \omega \in L^{2} .
$$


The weaker properties $A_{x} \omega \in L^{2}(x)$ for a.a.y, $A_{x} \omega \in L^{2}(y)$ for a.a. $x$ and corresponding statements for $B_{y} \omega$ follow from (29), resp. (30).

\section{REFERENCES}

1. T. Carleman, Sur les équations singulières a noyaux réelles et symétriques, Upsala. 1923.

2. J. von Neumann, Charakterisierung des Spektrums eines Integraloperators, Collected Works, Vol. 4, Pergamon, Oxford, 1962; 38-55.

3. H. Weyl, Rend. Circ. Mat. Palermo 27 (1909), 373-392.

4. B. Misra, D. Speiser and Gy. Targonski, Helv. Phys. Acta 36 (1963), 963-980.

5. A. C. Zaanen, Linear analysis, North-Holland, Amsterdam, 1956.

6. Gy. I. Targonski, Seminar on functional operators and equations, Lecture Notes in Mathematics, No. 33, Springer-Verlag, Berlin, 1962.

FORDHAM UNIVERSITY AND

Forschungsinstitut Für Mathematik der ETH, ZÜRICH 